\title{
Wayfinding Behavior within Buildings - An International Survey
}

\author{
ANAND VEERASWAMY, EDWIN R. GALEA, and PETER J. LAWRENCE \\ Fire Safety Engineering Group \\ The University of Greenwich \\ 30 Park Row, Greenwich, London SE10 9LS, UK
}

\begin{abstract}
A building wayfinding questionnaire study is presented which analyses the importance of a set of wayfinding criteria from a building evacuation perspective. The main path selection criteria tested in this questionnaire are handedness and length of the first leg of the path. The study involved 1166 participants from 36 countries. The results suggest that the handedness, a genetic factor, and the side of the road people drive on, a cultural factor, exert a significant influence on path choice. The results of this study clarify misconceptions existing in urban wayfinding studies regarding the importance of the length of the first leg of a path. Path selection criteria along with their relative rankings are suggested for inclusion in wayfinding algorithms used within evacuation models. It is not clear how large an effect these influences will have on evacuation analysis. This will be examined by introducing these factors into a new wayfinding algorithm recently introduced into the buildingEXODUS evacuation model.
\end{abstract}

KEYWORDS: wayfinding, escape routes, path selection, human behavior, human factors.

\section{INTRODUCTION}

Within the building environment, wayfinding describes the process by which an individual located within a complex enclosure decides on a path or route in order to reach a goal location. Within the building evacuation context, wayfinding describes the process in which the individual attempts to find a path which leads them to relative safety, usually the exterior of the enclosure. In most evacuation modeling tools, the process of wayfinding is either ignored or grossly simplified. In a recent review of 30 evacuation models wayfinding features were only mentioned in the context of two models [1]. On the whole, evacuation models assume that the simulated agents have complete knowledge of the structure and so follow a potential or distance map to their nearest exit - essentially selecting the path of minimum travel distance. Some models may even assume that a proportion of the occupants have partial knowledge of the structure and so are familiar with only some of the exits [2,3]. At least one model incorporates agent interaction with signage allowing agents completely unfamiliar with the structure to follow a signage chain leading to an exit [2,3]. Recently, there has been some effort to incorporate a modified form of urban wayfinding criteria within building evacuation models [4]. However, this may be questionable as the wayfinding process within buildings may be different to that within urban environments. If our computer models are to accurately represent the wayfinding process adopted by humans during building evacuation we must first understand how humans wayfind within such environments. Key to this is developing an understanding of the criteria used by humans in deciding which path to take.

The lack of sophistication in the manner in which wayfinding is treated within building evacuation models is due in part to the general lack of detailed knowledge concerning wayfinding within complex building layouts. While there are numerous wayfinding studies in urban environments [5-8], there is very little wayfinding research performed within complex building environments. Due to the difficulty in conducting wayfinding research, especially research which involves international participants, most of the research that is conducted makes use of surveys, questionnaires or virtual environments.

Golledge's urban wayfinding study [5,6] involved a sample of 32 adults, 16 male and 16 female. The sample was mostly students, with half the population being trained in geography. The participants in Golledge's study were asked to identify the path they would take on a map to get from an identified starting point to an identified end point. The maps used varied from uniform grids, to uniform grids with diagonals and grids with curved paths plus blockages. Participants were then asked to rank the criteria they used in selecting their path using a seven point scale; participants selected the criteria from a list of 10 provided. The criteria selected by the participants, in order of preference (with mean rating shown in brackets) are: shortest distance (4.2), least time (4.1), fewest turns (3.6), most scenic/aesthetic (3.5), first noticed (2.5), 
longest leg first (2.3), many curves (2.3), many turns (1.8), different from previous (1.8) and shortest leg first (1.7).

Conroy has studied route choice decisions made at consecutive road junctions over the duration of a single journey [7]. The study involved a virtual urban environment containing a variety of urban block shapes and involved 30 participants, 20 males and 10 females with a mean age of 28 . The geometry was the equivalent of $650 \mathrm{~m} \times 650 \mathrm{~m}$ and would take approximately $8.5 \mathrm{~min}$ to cross diagonally walking at an average pace. Participants spent on average 10 minutes immersed in the virtual world. The participants were instructed to enter the geometry at the top corner and make their way to the diagonally opposite bottom corner. From an analysis of the paths chosen, Conroy concludes that route selection is a competition between the desire to select the simplest route (i.e. straightest route to the destination) and the desire to maintain a heading closest to the direction of the destination from the origin (most direct route). Conroy goes on to suggest that the finding of Golledge in which people prefer to select the longest leg first (i.e. starting the journey by selecting the route option that has the longest line of sight) over the shortest leg first [5,6], is a result of the most direct route winning over the simplest route.

The study of Scharine and McBeath [9] investigated the choice people make in taking a left or right turn when all other conditions were equal. Their experiment involved 112 participants who were library patrons, 82 from the USA and 30 from the UK. The sample consisted of $87 \%$ right-handed people. Participants were asked to retrieve an object which was hidden at the end of an aisle formed by a shelf of books. The participants had to walk down a $10 \mathrm{~m}$ long corridor formed by book shelves and then turn either into the left aisle or the right aisle to retrieve the object. They found that $66 \%$ of the right-handed sample preferred to turn right while only $33.3 \%$ of the left-handed sample preferred to turn right. This indicates that the handedness of the participant is a strong indicator of the direction in which the person is likely to turn. In addition, $67.1 \%$ of the sample who drive on the right side preferred to turn right while $46.7 \%$ of the sample who drive on the left-side prefer to turn right. This indicates that while the side of the road that you drive on has an influence, it is not as strong as the handedness. Furthermore, it appears that handedness and driving side are additive factors, with approximately $70 \%$ of right-handed, right-side driving sample preferring to turn right, while approximately $48 \%$ of the right-handed, left-driving sample prefer to turn right. The sample size of left-side drivers was considered too small to draw definitive conclusions. This study would suggest that there is both a genetic component to wayfinding (handedness) and a cultural component (driving side).

While these studies indicate that there are a number of factors that influence wayfinding, the studies are either drawn from too small a sample or are not directly related to wayfinding within buildings. Furthermore, the study of Golledge, conducted in the USA and Conroy, conducted in the UK, do not consider handedness or driving side of the participants and the likely impact this may have on wayfinding. To address these issues and identify the factors which impact wayfinding within building environments, the authors have undertaken two large-scale international surveys. The first survey explored factors associated with; handedness, driving side, and preference for longest leg first (LLF) or shortest leg first (SLF). The second survey was designed to explore a wider range of potential factors which influence wayfinding. The results from the first questionnaire are presented in this paper. Once the factors which influence wayfinding within building are established, they will be incorporated within the wayfinding algorithm established in [4].

\section{THE WAYFINDING QUESTIONNAIRE}

Participants were invited to complete an on-line questionnaire. The call for participation to complete the on-line survey was undertaken via several different media e.g. website link, leaflet distribution, online forums, email mailing lists, friends/family/colleagues, a national appeal broadcast on BBC radio, snow balling, etc. Whilst the survey is currently on line (http://fseg.gre.ac.uk/wayfinding/index.asp), at the time of writing this paper, the results presented represent data collected from approximately July 2009 to July 2010. The survey consisted of three sections and required approximately 10-15 min to complete.

The first part consisted of five separate wayfinding tasks where the participants were shown five different hypothetical building layouts in turn (see Fig. 1), each with two paths, labeled A and B, leading to an exit. The participant was provided with written information stating that each path was equal in length and that they were completely familiar with the building layout. On selecting the preferred path, the participant was 
then asked to rate both paths on a scale of 1 to 6 where 1 is highly undesirable, 2 is very undesirable, 3 is a little undesirable, 4 is a little desirable, 5 is very desirable and 6 is highly desirable. On completing this, the participant was then asked to imagine that they were now in an emergency evacuation situation in the same building and choose their preferred exit path. For the emergency evacuation option, the participant was informed that both routes were considered safe and free of smoke or other fire hazards.

Question 1 is intended to investigate the participant's preference for selecting the left-hand or right-hand path (see Fig. 1a). In the second (see Fig. 1b) and third (see Fig. 1c) questions, the building geometries either have the LLF path on the left (see Fig. 1b) or right (see Fig. 1c). The LLF path is also the most direct path having the least bearing to the exit. In order to remove 'the most direct path' factor from consideration, Questions 4 (Fig. 1d) and 5 (Fig. 1e) were designed where the start position is in line with the exit, thus both paths have an equal bearing to the exit. Initially the length of the paths in Questions 4 and 5 were kept the same. However a pilot study indicated that people perceived the angled path, Path B in Question 4 and Path A in Question 5 (Fig. 1d and Fig. 1e), to be slightly longer in spite of being reminded that both paths are of the same length. Hence the angled paths were made slightly shorter in order to compensate for this visual illusion.

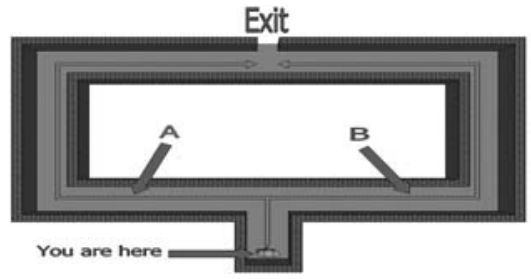

(a)

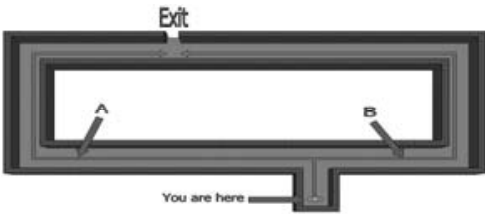

(b)

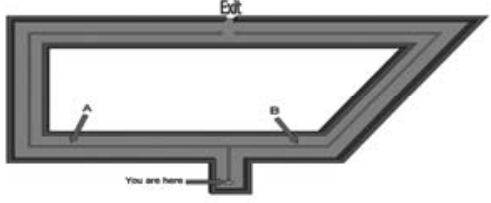

(d)

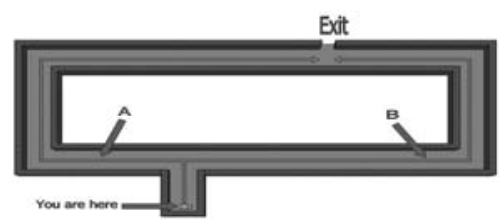

(c)

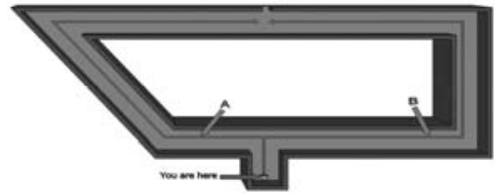

(e)

Fig. 1. Path selection tasks in the questionnaire.

In the second part of the survey the participants were given a list of factors and were asked to choose those that influenced their wayfinding decisions in the first part of the survey. Participants could select more than one option and were also given the opportunity to list other factors that influenced their decision. The list of factors provided was:

- I prefer to take the path with the longest leg first

- I prefer to take the path with the shortest leg first

- I have a preference for paths on my left

- I have a preference for paths on my right

- I have a preference to take the clockwise paths

- I have a preference to the take the anticlockwise path

- I choose the path which appeared to be the most direct

- I choose randomly

The third part of the survey involved collecting participant demographic information. Two pilot studies were conducted to check the general level of understanding of the questions. A few modifications were made to the questionnaire based on the pilot studies and this data was not included in the overall questionnaire results. 


\section{THE SAMPLE POPULATION}

A total of 1200 participants from 36 countries took part in the survey of which 1166 were considered eligible to take part in the analysis. The countries with the largest response to the survey are: UK, 649 (56\%); USA, 113 (10\%); Australia, 72 (6 \%); India, 62 (5\%); Germany, 46 (4 \%) and Netherlands, 25 (2\%). Of this sample, a total of 336 (29\%) of the participants drive on the right-side of the road e.g. USA, Germany, Netherlands, etc, and 830 (71 \%) drive on the left-side of the road e.g. UK, Australia, India, etc. Furthermore, 1010 (87 \%) of the participants are right-handed while 156 (13\%) are left-handed. Our sample of left-handed people is equivalent to the world average of 10-13\% of the population being lefthanded [10]. Of the sample population who drive on the left-side of the road (830), 728 (88\%) are righthanded (note, the right-handed left-driving sub-population is referred to as RHLD). Of the left-driving sample, 102 (12\%) are left-handed (note, the left-handed left-driving sub-population is referred to as LHLD). Of the sample that drive on the right-side of the road (336), 282 (84\%) are right-handed (referred to as RHRD) while 54 (16 \%) are left-handed (is referred to as LHRD).

\section{THE MAIN RESULTS AND DISCUSSION}

The findings from Part 1 of the study are first presented, followed by the findings from Part 2 . The Part 1 results are first summarized and then the responses to each of the wayfinding questions are examined in turn. It is important to note that the results for the path selection under normal conditions were virtually identical to those under emergency conditions and so only the results under normal conditions are presented. All the conclusions presented in this paper are statistically significant using the Chi-Square test with Yates [11] correction.

\section{Discussion of Part 1 Results}

In this section the results from the path selection tasks for Questions 1-5 are first summarized. This is then followed by a detailed investigation of the results from each path selection task. The results for each path selection exercise are examined in detail with a focus on: Question 1 - impact of the participants handedness and driving side on the path selection; Questions 2 and 3 - impact of the length of the first leg on path selection (together with participant handedness and driving); Questions 4 and 5 - as for Questions 2 and 3 but with the path directness removed as an influencing factor.

\section{Overview of Path Selection Results}

A summary of the path choice made by the entire sample population (1166) for each of the five questions is shown in Fig. 2. In the first four wayfinding tasks (Questions 1-4), the participants clearly have a preference for right-hand paths which is statistically significant at a 0.001 confidence level.

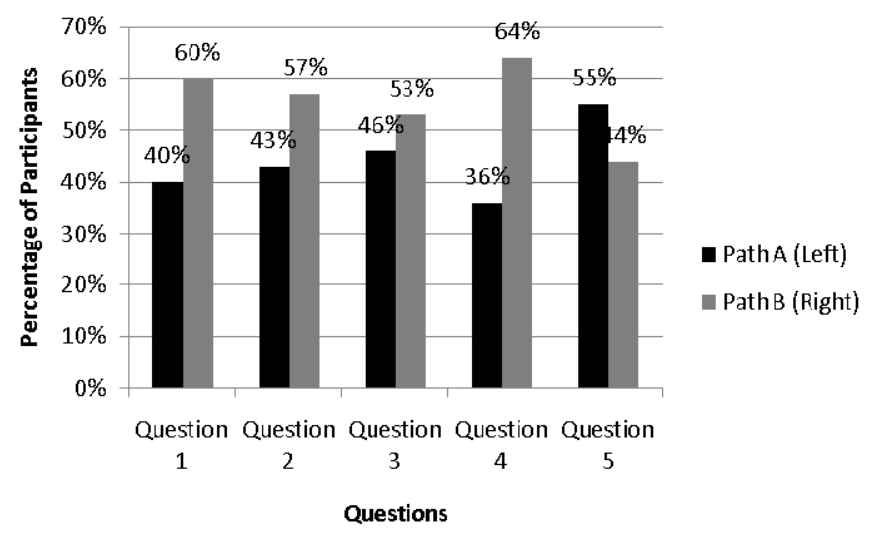

Fig. 2. Percentage of the sample population selecting Paths A and B in wayfinding tasks.

This strong preference for right-hand paths is examined in more detail in the following sections. These results suggest that on the whole people generally have a slight preference for right-hand paths. However, 
in the fifth wayfinding task there is a significant preference for the left-hand path. The reasons for this apparent difference in path selection are examined in the next sections.

\section{Question 1: The Influence of Handedness on Path Selection}

In the first question, both path options were identical, the only variable that was different was that one path was on the left and the other path was on the right. Taken across the entire sample (1166), $60 \%$ (700) chose the right-hand path and $40 \%$ (466) chose the left-hand path (see Fig. 3). The chi-square goodness of fit test [11] $\left(\chi^{2}=42.39\right)$ suggests that this distribution is valid at a 0.001 level of significance. Thus people in general are more likely to turn right than left.

In the right-handed sub-population (1010), 64 \% (646) chose the right-hand path (see Fig. 3). Considering that $87 \%$ of the entire sample is right-handed, it is not surprising that the increase in the preference for right-hand paths is as small as $4 \%$ compared to the entire sample. In the left-handed sub-population (156), $71 \%$ (111) have chosen the left-hand path and $29 \%$ (45) have chosen the right-hand path (see Fig. 3). This distribution is also valid at a 0.001 level of significance $\left(\chi^{2}=23.52\right)$ which implies that left-handed people have a strong preference for left-hand paths. Thus handedness of people is a strong factor which determines people's preference for right/left-hand paths.

A chi-square test of independence was also performed to check if the right/left-hand paths chosen by people are independent of the handedness of the participants. The null hypothesis is that the path choice of people is independent of their handedness. The paths chosen by the right-handed sub-population and the left-handed sub-population have been considered for this analysis. The chi-square result $\left(\chi^{2}=60.69\right)$ suggests that the null hypothesis can be rejected at a 0.001 significance level. Thus handedness does have a very strong influence on the right/left-hand path choice.

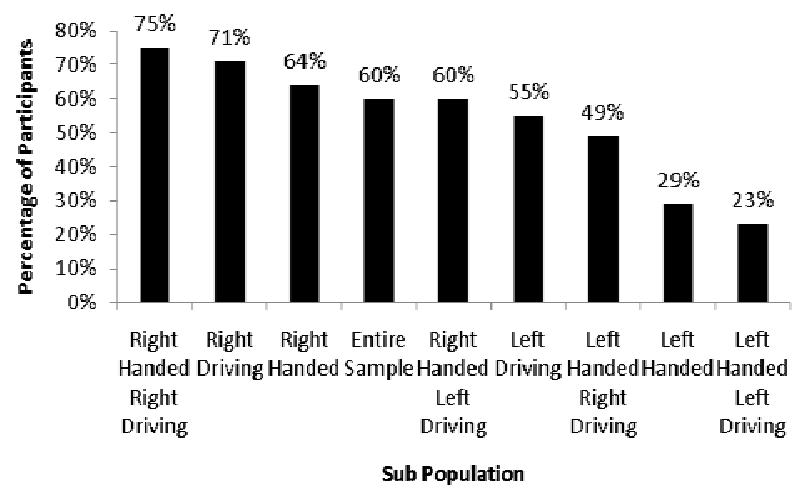

Fig. 3. Percentage of each sub-population choosing right-hand paths.

\section{Question 1: The Influence of the Driving Side on Path Selection}

In the right-side driving sub-population (336), $71 \%$ (239) have chosen the right-hand path (see Fig. 3). People driving on the right-side of the road have a strong preference to right-hand paths at a 0.001 level of significance $\left(\chi^{2}=57.26\right)$. In fact, people driving on the right-side of the road have a stronger preference to right-hand paths than the right-handed sub-population where only $64 \%$ chose the right-hand paths (see Fig. 3). This is because the majority (84 \%) of the right-side driving sub-population is also right-handed. This suggests that in addition to handedness, the driving side also influences the choice of right-hand paths in a complementary (additive) fashion. In the left-side driving population (830) $55 \%$ (457) have chosen the right-hand path (see Fig. 3). The right-hand path preference has fallen by $5 \%$ compared to the entire sample which suggests that driving on the left-side influences people to choose left-hand paths. However, $88 \%$ (730) of the left-side driving sub-population is right-handed which explains why right-hand path is still popular. In the case of the left-side driving sub-population, right-handedness and left-side driving are in opposition, reducing the overall number of people who select the right-hand path. This suggests that handedness of people (genetic factor) is a more influential factor than the driving side (cultural factor) when it comes to influencing wayfinding path choices. 
A chi-square test of independence was also performed to check if the right/left-hand paths chosen by people are independent of the driving side by considering the right-side driving and left-side driving subpopulations. The chi-square result $\left(\chi^{2}=24.31\right)$ suggests that the driving side has a strong influence on the right/left-hand paths chosen at 0.001 significance level. It is interesting to note that even though the majority in the right-side drive and left-side drive sub-populations has preferred the right-hand path, the driving side still has a significant influence on the path choice.

\section{Question 1: The Influence of the Handedness and Driving Side on Path Selection}

Clearly, the handedness and driving side are factors which each have a significant influence on path selection. In this section the influence of a combination of the two factors is considered. Figure 3 shows the percentage of each sub-population choosing right-hand paths in the first question of the questionnaire relating to the geometry in Fig. 1a. The sub-populations are arranged in descending order of preference for right-hand paths from left to right. Thus the sub-populations having a higher preference for right-hand paths are on the left and the sub-populations having a lower preference for right-hand paths are on the right. Clearly, right-handed people driving on the right-side have the strongest preference for right-hand paths and the left-handed people driving on the left-side have the lowest preference for right-hand paths (and the highest preference for left-hand paths). The preference for right-hand paths in the first six sub-populations from the left is statistically significant at the 0.001 confidence limit. The preference for left-hand paths for the last two sub-populations is also statistically significant at the 0.001 confidence limit.

The right-handed sub-population has a strong preference for right-handed paths at $64 \%$. The preference for right-hand paths increases to $75 \%$ in the RHRD sub-population. This suggests that the preference for righthand paths is amplified if one is right-handed and from a country driving on the right side of the road compared to when one is simply right-handed. The left-handed sub-population has little preference for right-handed paths at $29 \%$. The preference for right-hand paths further decreases to $23 \%$ for the LHLD sub-population. This suggests that the preference for left-hand paths is amplified if one is left-handed and from a country driving on the left side of the road compared to when one is simply left-handed.

The RHRD sub-population has a $15 \%$ greater preference for right-hand paths than the RHLD subpopulation. Thus if one is right-handed and driving on the left side of the road then the preference for righthand paths while high is not as high as it is when one is right-handed and driving on the right-side of the road. The LHLD sub-population exhibits a $25 \%$ greater preference for left-hand paths than the LHRD subpopulation. This suggests that if one is left-handed and driving on the right-side of the road there is a small preference for left-hand paths, and if one is left-handed and driving on the left side of the road there is a strong preference for left paths. The LHRD sub-population has a $51 \%$ preference for left-handed paths, close to a 50-50 preference for right- and left-hand paths. The small preference for left-handed paths for this sub-population may be attributed to the small number of participants in this category, only 54, with the next smallest category having 102 participants (LHLD). It is expected that if the number of participants in the LHRD category were increased, a stronger preference for left-handed paths would emerge for this subpopulation. However, according to Cochran [12], the expected and observed frequencies in this subpopulation are sufficient to make statistically significant conclusions.

Considering that the RHLD sub-population has a strong preference for right-hand paths and the LHRD subpopulation has a slight preference for left-hand paths, one can deduce that handedness of people is a stronger factor than the driving side in influencing the right/left-hand path choice. The same deduction can also be made comparing the $\chi^{2}$ values for the handedness and driving side analyses. The $\chi^{2}$ value for the influence of handedness, 60.69 , is greater than the $\chi^{2}$ value for the influence of the driving side, 24.31. This supports the conclusion that handedness (a genetic factor) is a more influential factor than the driving side (a cultural factor) in affecting path choice. Furthermore, if the driving side is the same as the handedness, these factors reinforce each other however, if they are opposite, they work in opposition.

\section{Questions 2 and 3: Length of the First Leg of the Path}

In Questions 2 (Fig. 1b) and 3 (Fig. 1c), the length of the first leg is introduced as a path choice factor. In Question 2 the right-hand path is the shortest leg while in Question 3 the right-hand path is the longest leg. Figure 4 shows the percentage of each sub-population that selected the right-hand paths in Questions 2 and 3. 
It is interesting to note that in Questions 2 and 3, the sub-populations containing a majority of righthanders, which are the first six columns from the left of Fig. 4, have all shown a significant preference for the right-hand path regardless of whether it is the SLF/LLF path. A similar trend is found for the subpopulations consisting of left-handed people, which are the last two columns on the right in Fig. 4, namely a significant preference for the left-hand path regardless of whether it is the SLF/LLF path. The exception to this is the LHRD sub-population who prefer the right-hand path - however, as suggested earlier the sample size may be too small to make meaningful comparisons. The preference for right- or left-hand paths regardless of the length of the first leg suggests that for the majority of the population the handedness of the path is a more influential factor than the length of the first leg in affecting path choice.

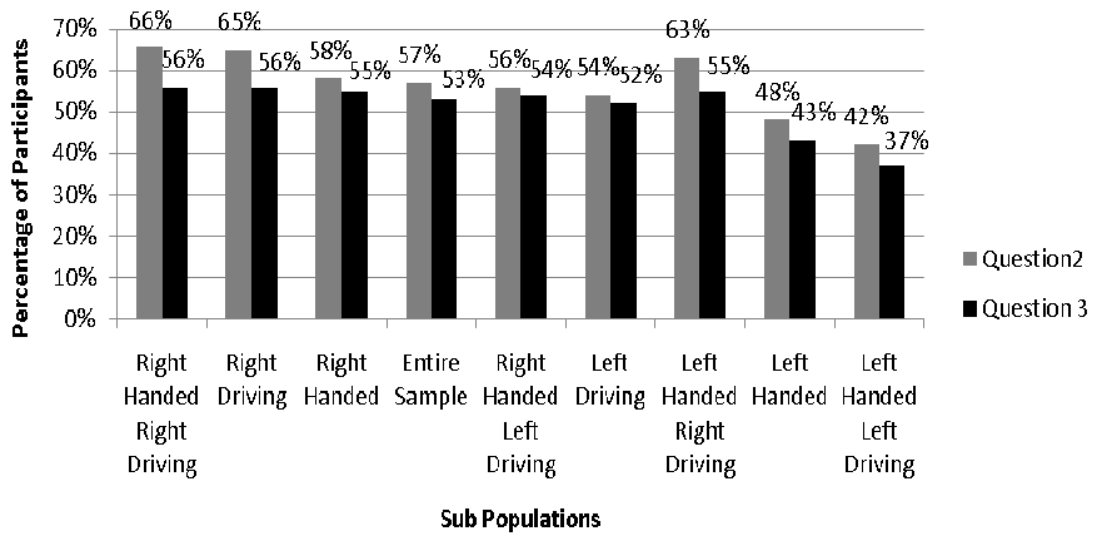

Fig. 4. The percentage of each sub-population choosing the right-hand path in Questions 2 and 3.

However, for some of the population, selecting the SLF or the LLF will be more important than the handedness of the path. Consider the sub-populations that prefer the right-hand path i.e. the first six columns of the left of Fig. 4, when the right-hand path preference is compared to that of Question 1 (see Fig. 3) it is noted that fewer people select the right-hand path when the first leg of the path is short or long. For example, consider the RHRD sub-population for which the preference for selecting the right-hand path is strongest with $75 \%$ of the sub-population preferring the right-hand path in Question 1. For some of this sub-population, selecting the SLF or the LLF will be more important than selecting the right-hand path resulting in the proportion of the sub-population falling below $75 \%$ in Questions 2 and 3.

It is noted from Questions 1 and 2 that $9 \%$ (75-66 \%) of the RHRD sub-population that would have been expected to select the right-hand path have selected the LLF path (i.e. the left-hand path) in preference. For this small group, selecting the path with the LLF is more important than the handedness of the path and so they have selected the left-hand path. Similarly, as some of this sub-population will have a stronger preference for the SLF than the right-hand path, the percentage of people selecting the right-hand path in Question 3 will be less than $75 \%$. It is also noted that $19 \%$ (75-56 \%) of the RHRD sub-population have selected the SLF in preference to the right-hand path. For this group, selecting the path with the SLF is more important than the handedness of the path and so they have selected the left path.

Furthermore, $66 \%$ of the RHRD sub-population have selected the SLF path when it is the right-hand path (Question 2) while $56 \%$ of the sub-population have selected the LLF when it is the right-hand path. This suggests that selecting the SLF is a more dominant secondary factor than selecting the LLF. Within the RHRD sub-population, more people prefer the right-handed path when it is also the SLF path than when it is the LLF path.

Similar trends are found for the sub-populations that prefer the left-hand path i.e. the last two columns on the right of Fig. 4. When the left-hand path preference is compared to that of Question 1 (derived by taking $100 \%$ - right-hand preference (\%) shown in Fig. 3) it is noted that fewer people select the left-hand path when the first leg of the path is short or long. For example, consider the LHLD sub-population for which the preference for selecting the left-hand path is strongest with $77 \%(100-23 \%)$ of the sub-population preferring the left-hand path in Question 1. For some of this sub-population, selecting the SLF or the LLF 
will be more important than selecting the left-hand path resulting in the proportion of the sub-population falling below $77 \%$ in Questions 2 and 3.

It is noted that from the $77 \%$ who chose the left-hand path in Question 1 only $63 \%$ (100-37\%) have chosen the left-hand and SLF path in Question 3. Hence $14 \%$ (77-63 \%) of the LHLD sub-population that could normally have been expected to select the left-hand path have selected the LLF path (right-hand path) in preference. For this group, selecting the path with the LLF is more important than the handedness of the path and so they have selected the right-hand path. Similarly, as some of this sub-population will have a stronger preference for the SLF than the left-hand path, the percentage of people selecting the left-hand path in Question 3 will be less than $77 \%$. It is noted that $19 \%$ of the LHLD sub-population have selected the SLF in preference to the left-hand path. For this group, selecting the path with the SLF is more important than the handedness of the path and so they have selected the right-hand path.

Furthermore, $63 \%$ of the LHLD sub-population have selected the SLF path when it is the left-hand path (Question 3) while $58 \%$ of the sub-population have selected the LLF path when it is the left-hand path (Question 2). This suggests that selecting the SLF is a more dominant secondary factor than selecting the LLF. Within the LHLD sub-population, more people prefer the left-handed path when it is also the SLF path than when it is the LLF path. The same trend is also found across the other sub-populations and the entire sample.

From the results of Questions 1-3 we conclude that for the majority of people, the handedness (a genetic factor) is the most important factor in influencing a person's path choice. The handedness however is influenced in turn by the driving side (a cultural factor) - if the driving side is the same as the handedness, these factors reinforce each other while if they are opposite, they work in opposition. The next most important factor influencing path selection is the length of the first leg of the path. The preference for SLF paths is greater than the preference for LLF paths. This result is interesting as it contradicts the conclusions of urban wayfinding research which suggests that the preference for LLF is greater than that for the SLF [5-7]. Finally, for a small proportion of the population, selecting the SLF or the LLF will be the most important factor in influencing a person's path choice, with selecting the SLF being more dominant that selecting the LLF.

On completing the Question 2 and 3 path selection tasks, participants were asked to comment on why they selected a particular path (see Fig. 5). This was an open question with no prompts provided. Each of these comments have been analyzed and generalized into certain common factors. For example the following comment was made by a participant, “... this was my first reaction, I tend to turn to my right ...”. This was inferred to mean that the participant had a preference for right-handed paths. A comment made by another participant was, "Initial path looks to be shorter". This was inferred to mean that the participant had a preference for the SLF path. From Fig. 5 it is noted that the SLF criterion is identified as being more important to most people than the LLF path.

It is somewhat surprising that the SLF criterion has been mentioned by more people than the right-handed path criterion. However, among the group who claim to have selected paths based on instinct, 64 had selected the right-handed path (and 45 the left-handed path). If these are added to the right-hand path selection, then the right-hand path selection dominates. Furthermore, $18 \%$ and $23 \%$ of the participants who answered Questions 2 and 3 respectively did not provide a reason for their path choice. Here again, at least half of these participants will have selected the right-hand path, but it is not clear if they selected the right-hand path because it was the right-hand path or if it had the SLF. Thus it is not clear whether righthanded paths or SLF paths exert the most influence on path selection based on the results from this question.

In addition, right-handed paths have been mentioned by more participants than left-handed paths which is consistent with the path selection tasks. The number of participants who claim that they made a random path selection is very small, representing only $2 \%$ of the responses to Questions 2 and 3 . Finally, the number of participants who suggested that they made a path selection based on the most direct route was only $7 \%$ of the responses to Questions 2 and 3 . While it is not clear what the participants meant by this statement, the results suggest that consciously selecting a path on the basis of its directness appears to exert a low influence on path selection. 


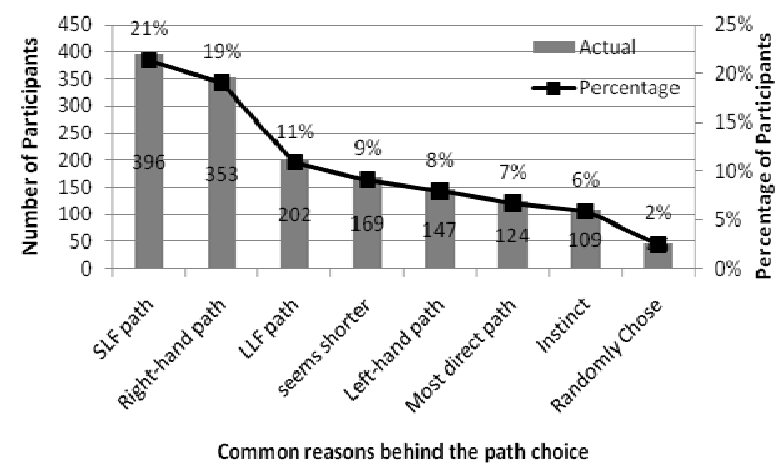

Fig. 5. Participant path selection comments for Questions 2 and 3.

\section{Questions 4 and 5: Removing Directness from Path Selection}

Question 4 (Fig. 1d) and Question 5 (Fig. 1e) investigate the same factors as Question 2 and 3 with the exception that the LLF path is no longer the most direct path. By positioning the exit directly opposite the starting point, both the LLF and SLF path are considered equally direct as both paths have the same bearing on the exit. Furthermore, the travel distance associated with each path option is still equal as in the other cases. As a result of removing the path directness from consideration while maintaining equal total travel distances, the SLF path has acquired a sharp angle turn. The percentage of each sub-population selecting the right-hand path in Questions 4 and 5 is shown in Fig. 6. As was found in Questions 2 and 3, the righthanded path which is also the SLF path (Question 4) is preferred by more people than the right-hand path which is also the LLF path (Question 5). Furthermore, the majority of people in Question 4 prefer the righthanded path (which is also the SLF path) however, unlike in Question 2, this trend extends to all of the subpopulations, even those who are expected to have a preference for left-handed paths i.e. the last three subpopulations on the right side of Fig. 6.

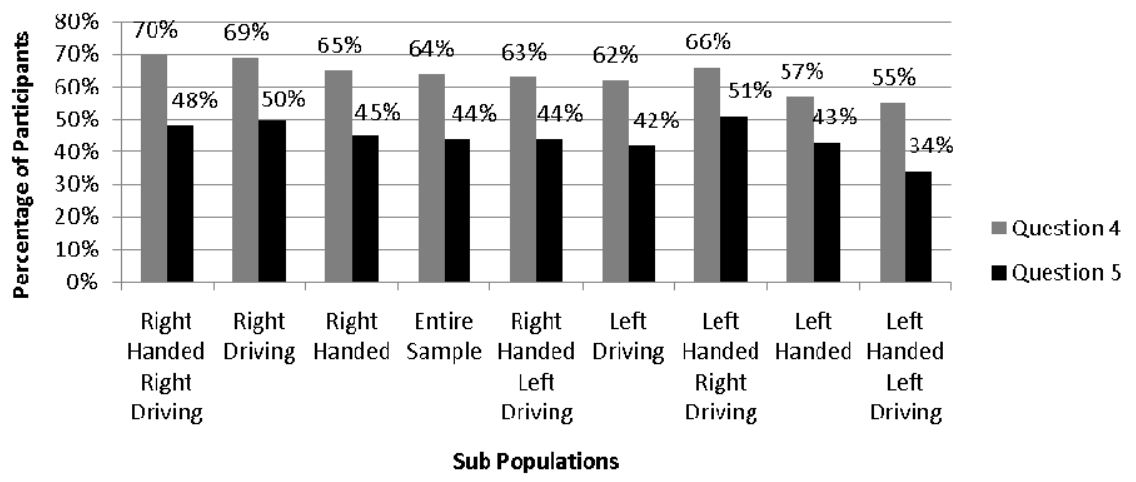

Fig. 6. The percentage of each sub-population choosing the right-hand path in Question 4 and 5.

A result which is counter to expectations is that the majority of people in virtually all the sub-populations prefer the left-handed path (which is also the SLF paths) in Question 5. This even includes those subpopulations who are expected to have a preference for right-handed paths. This rejection of the right-hand path by the majority of people in Question 5 may be a result of the strong preference for the SLF path however, if this were simply the case, a similar trend would have been expected in Question 3.

As with Questions 2 and 3, it can again be concluded that the majority of people prefer the SLF paths compared to the LLF paths. However, the preference for SLF paths appears to be more significant in Questions 4 and 5 than in Questions 2 and 3, so much so that in Question 5, the majority of people have selected the left-hand path in preference to the right-hand path. There are two possible explanations for the 
increased trend in the preferences for the SLF path over the LLF path. Either the LLF path has become less attractive or the SLF path has become more attractive.

In an attempt to resolve this question, consider the nature of the paths in Questions 2 and 3 and those in Questions 4 and 5. There are two key differences between the paths in Questions 4 and 5 and those in 2 and 3. The first is that the initial leg of each path in Questions 4 and 5 is such that the bearing of each path to the exit is identical. Thus each path appears equally direct, whereas in Questions 2 and 3, the LLF path was the more direct path. By removing the path directness aspect from consideration, between $3 \%$ and $13 \%$ more people across all the sub-populations have selected the SLF path in Question 4 compared with Question 2. If this reasoning is correct, this would suggest that for between $3 \%$ and $13 \%$ of the population, selecting the most direct path will be the most important factor in influencing a person's path choice. For this group of people, when the most direct path option is removed, the LLF path (which corresponds to the most direct path) is no longer considered attractive and so the SLF path is selected.

The second difference is that in Questions 4 and 5, the SLF path has a sharp angle turn. Thus an alternative explanation for the increased popularity of the SLF path is that for between $3 \%$ and $13 \%$ of the population, selecting a path with a sharp angle turn is the most important factor in influencing a person's path choice. While both these explanations are plausible, neither is particularly convincing. By simply considering the path that was selected it is not possible to convincingly resolve the issue as to why the SLF path appears to be more attractive in Questions 4 and 5 compared to Questions 2 and 3. However the results of the open questions following Questions 4 and 5 provide some insight into the rationale used by participants in selecting their preferred path (see Fig. 7).

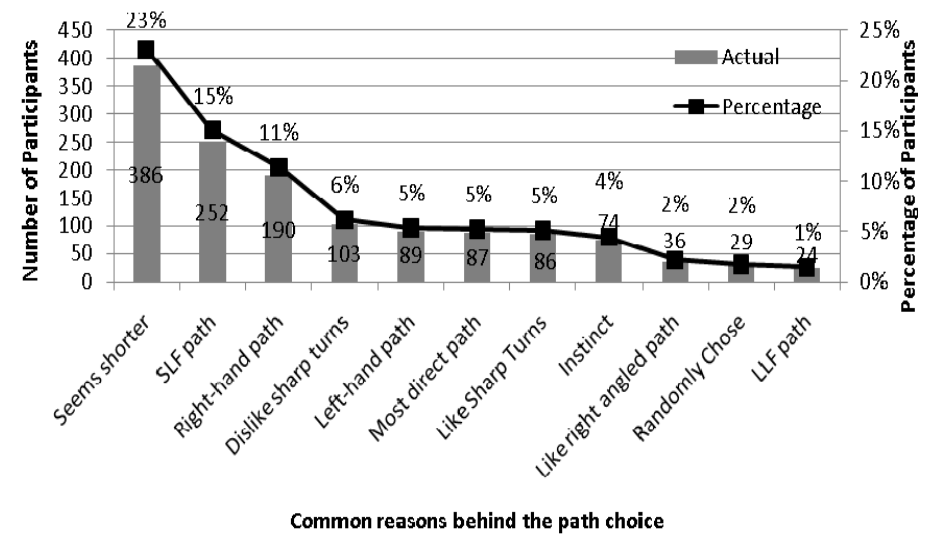

Fig. 7. Participant path selection comments for Questions 4 and 5.

A total of 1671 comments were provided by the participants to Questions 4 (851 comments) and 5 (820 comments). Of these, $19 \%$ (315) provided answers that could not be classified or were in groups that consisted of less than 20 responses or were unclear. Of the comments received, $23 \%$ (386) identified that they selected what they thought was the shorter path, even though they were informed that both paths were the same length. Furthermore, of these people, almost three times as many (296) selected the path with the sharp angle turn, i.e. the path with the SLF. This explains why there was an increase in the number of people who selected the SLF path in Questions 4 and 5 i.e. a large number of people selected the SLF path because they thought this path also had the shorter total length. The reasons suggested previously concerning the most direct path and the sharp angle, while plausible clearly do not correlate with the reasons provided by the participants for their path selection. This demonstrates that interpreting why people have selected a particular path based only on the paths selected can result in incorrect conclusions being drawn. Furthermore, $6 \%$ (103) of the participants have indicated that they selected their particular path because they disliked paths involving sharp angle turns while $5 \%$ (86) selected their path because they liked paths involving sharp angles. In addition, $5 \%$ (87) claimed that they selected their path because it was the most direct, even though both paths had the same heading relative to the exit. Clearly, the term 'most direct' is open to interpretation and may not mean the same thing as defined earlier in this paper. 
The comments also support the observation that the SLF criterion is more influential than the LLF criterion in influencing path choice and that right-handed paths are more influential than left-handed paths. As with Questions 2 and 3, the SLF path appears to be more influential than the right-handed paths. However, of the group that selected their path based on instinct, 43 selected the right-hand path. If these are then added to the group that selected the right-handed path, then the SLF and right-handed paths have approximately the same level of influence on path selection. Furthermore, the number of people who selected the SLF path is inflated due to a substantial number of people selecting this path because they thought the entire path was shorter due to the sharp angled corner.

\section{Discussion of Part 2 Results}

On completion of the wayfinding tasks, participants were asked to identify the factors which influenced their wayfinding decisions. Unlike the open questions following each wayfinding task in which participants were asked to provide a reason for their path choice, they were given a list of factors to choose from and more than one factor could be selected. Presented in Fig. 8 are the results from this question. In total (1150) $99 \%$ of the participants completed this section, making 2309 selections. On average, each participant selected 2.0 criteria. The wayfinding criteria are arranged in descending order with the most popular criteria being on the left and the least important criteria being on the right. It is noted that the criterion 'Most Direct' was selected by $24 \%$ (547) of the participants and so is the criterion which most influenced path selection. This is considered surprising as it did not figure highly in Questions 2 to 5, indeed it was only mentioned by $7 \%$ of the participants in Questions 2 and 3 and $5 \%$ in Questions 4 and 5. Furthermore, as shown in Questions 4 and 5, both paths had the same initial bearing on the exit and so could be considered equally direct. Within the context of this survey, it is suggested that not all the participants had a clear or the same understanding of the term 'most direct'. The term can clearly be interpreted to mean different things to different people. However, the result clearly indicates that participants have a strong preference for paths which appear to them to be the most direct, although it is not clear how they define this term. As the meaning of the term 'most direct' is considered to be ambiguous within the context of this analysis, it is excluded from further consideration.

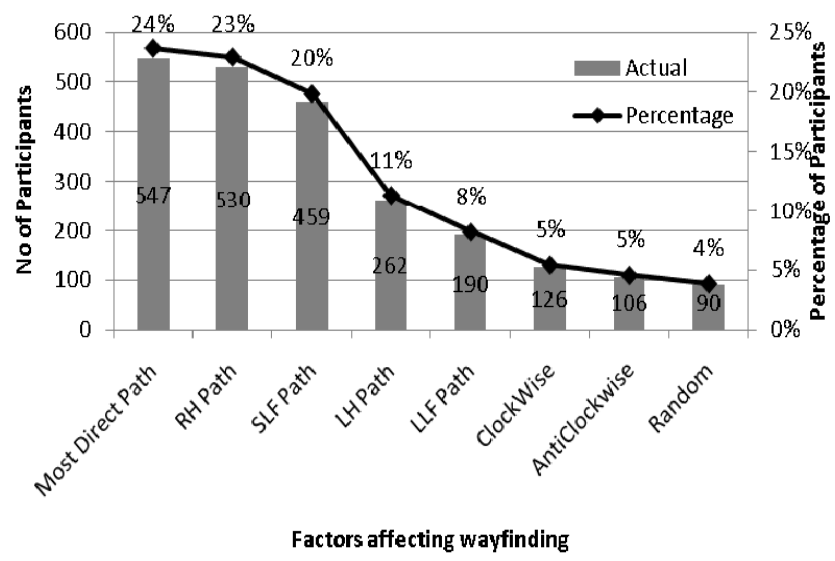

Fig. 8. Participant identified factors which influenced their path selection decisions.

Excluding the responses identifying the 'most direct' path, the main results support the findings of the path selection tasks, notably selecting a right-handed path (23\%) appears to be a marginally more important criteria for most people than the SLF path criterion (20\%). Furthermore, the SLF path criterion is more than two times more influential than the LLF path criterion and the right-handed path criterion is more than two times more influential than the left-handed path criterion. It is noted that the magnitude of the differences between preference for left/right and SLF/LLF paths in this part of the survey is significantly greater than suggested by the findings of the wayfinding tasks. Also, as approximately $5 \%$ of the responses indicated a preference for 'clockwise' or 'anticlockwise' paths, these criteria are not considered to be significant. Also, as only $8 \%$ of the returns indicate a random path selection, this suggests that the majority of the participants had reasons for their path selection. 


\section{RELATIVE IMPORTANCE OF THE WAYFINDING CRITERIA}

The results presented in Fig. 8 show the relative importance of the main wayfinding criteria as identified by the participants on completing the wayfinding tasks. The most influential criteria, in order of importance are selecting; right-handed paths (23\%), SLF paths (20 \%), left-handed paths (11 \%) and LLF paths (8\%). These rankings are based on the opinion of the participants and do not necessarily correlate with their actual path selection. To determine the relative importance of the path selection criteria, the results from Questions 2 and 3 are further investigated. The results from Questions 4 and 5 are being left out of this analysis due to uncontrolled factors such as perceived total path length and multiple factors such as like/dislike of sharp angle turns impacting the path selection. It is to be noted that these factors are examined in the second survey described earlier. The relative importance of the four path selection criteria is determined as follows: if a participant selected right-hand paths in both Questions 2 and 3 then they clearly prefer right-hand paths over all other criteria (i.e. left-hand paths, SLF, LLF); if a participant selects the left-hand paths in both Questions 2 and 3, then they clearly prefer left-hand paths over all other criteria; if a participant selects the SLF paths in both Questions 2 and 3, then they clearly prefer SLF paths; finally if a participant selects the LLF paths in both Questions 2 and 3, then they clearly prefer the LLF paths. All participants who have answered both Question 2 and 3 must fall into one of these four preference categories. These results for this analysis for various sub-populations are presented in Table 1.

Table 1. Summary of path preference criteria based on wayfinding tasks in Questions 2 and 3.

\begin{tabular}{|c|c|c|c|c|}
\hline $\begin{array}{c}\text { Sample } \\
\text { population }\end{array}$ & $\begin{array}{c}\text { RH path } \\
\text { preference (\%) }\end{array}$ & $\begin{array}{c}\text { SLF path } \\
\text { preference (\%) }\end{array}$ & $\begin{array}{c}\text { LLF path } \\
\text { preference (\%) }\end{array}$ & $\begin{array}{c}\text { LH path } \\
\text { preference (\%) }\end{array}$ \\
\hline Entire sample & 31 & 29 & 23 & 17 \\
\hline RH & 30 & 28 & 23 & 20 \\
\hline LH & 20 & 28 & 23 & 29 \\
\hline RD & 33 & 32 & 23 & 12 \\
\hline LD & 30 & 28 & 23 & 20 \\
\hline RHRD & 33 & 33 & 23 & 11 \\
\hline LHRD & 35 & 25 & 23 & 18 \\
\hline RHLD & 32 & 28 & 23 & 18 \\
\hline LHLD & 15 & 29 & 23 & 33 \\
\hline
\end{tabular}

If we compare the results for the entire sample in Table 1 with those from the Part 2 results (see Fig. 8) we note that there is broad agreement in the trends namely; right-handed paths are significantly more preferential than left-handed paths and right-handed paths are marginally more preferential than SLF paths. There is also agreement in that SLF paths are more preferential than LLF paths however, the results derived from the wayfinding tasks in Questions 2 and 3 suggest that the difference is marginal whereas the difference derived from the Part 2 results is significant. The results from the two analyses contradict each other in that, based on the results derived from Questions 2 and 3, LLF paths are significantly more preferential than left-handed paths (see Table 1) whereas, based on the Part 2 analysis (see Fig. 8), lefthanded paths are marginally more significant than LLF paths. Here we adopt the rankings based on the actual wayfinding tasks as being representative of human behavior within buildings as these were the results of actual wayfinding tasks as opposed to opinions.

The ranking of the path preference criteria based on the entire sample presented in Table 1 provide a means for ranking path preference in evacuation models. The results are significantly different from those generated by Golledge's urban wayfinding study $[5,6]$ in that the results from this study suggest that SLF paths are more preferential than LLF paths. These differences may be due to Golledge's results being based on an urban environment or due to the small and selective sample used in his study. This result is also different to that of Conroy [7] for similar reasons. Furthermore, the suggestion by Conroy that path directness is a significant path selection criterion [7] is not supported by the results of the wayfinding tasks in Questions 2 to 5 and when participants were asked to suggest why they selected their particular path. However, it was highlighted as the most significant criterion when the participants were asked to select path finding criteria from a list. Neither of these studies included the concept of handedness as a determinant of path selection. This study has shown it to be the most important factor. These results are 
similar to those of Scharine and McBeath [9] however, their study did not include the SLF and LLF factors and their sample was much smaller.

The results for the entire sample are intended to represent an average result which can be applied to the population as a whole. It is acknowledged that not every individual within a given population will exhibit the same type of behavior as suggested by the preference distribution for the entire sample. People are complex and wayfinding decision making is multi-faceted with a number of different influential factors driving decision making, some of which reinforce each other while others oppose each other. The nature in which these influential factors work may also be dependent on the nature of the individual, in some groups working together while in other groups working in opposition. Not everyone will be influenced in the same way by the influential factors that determine wayfinding behavior - unfortunately these are not universal truths! However, from a modeling perspective it is desirable to have a global ranking of the wayfinding criteria that can be applied to a population as a whole. Unfortunately, the ranking of path preference criteria presented in Table 1 shows that there are significant differences in the nature of the ranking distributions for important sub-populations. For example, a sample population made up of individuals from countries that drive on the left side of the road will display a significantly different wayfinding preference to a sample population made up of people from countries that drive on the right-side of the road. Furthermore, each of these populations can be further broken down into sub-populations of left-handed and right-handed people each of which display significantly different wayfinding preferences. Thus it is seen that genetic and cultural differences may influence our wayfinding preferences and thus our ability to evacuate efficiently.

It is suggested that in situations where the nature of the population is not clear or there will be a significant mixed population, the path preference ranking for the entire sample is used. If it is known that an application is primarily catering for individuals who drive on the left e.g. a UK application, then the leftside driving path preference is used whereas if the application is primarily catering for individuals who drive on the right e.g. a USA application, then the right-side driving path preference is used. Furthermore, these country specific can be further broken down into left-handed and right-handed sub-populations. While it may not be known which individual within the population is left-handed or right-handed, the international average of $13 \%$ of the population being left-handed can be applied. It is not clear how large an effect these differences in sub-population path preference criteria will have on evacuation analysis. This is currently being examined by introducing these factors into the recently developed wayfinding algorithm introduced into the buildingEXODUS evacuation model [4].

\section{CONCLUSION}

This work has identified factors which influence wayfinding decisions in building evacuation and normal circulation conditions. The results are based on an international survey involving 1166 participants from 36 countries. The survey involved participants completing path selection tasks based on five simplified corridor layouts. It was found that there was no significant statistical difference between the path selection criteria used in normal circulation and evacuation. A main result from this study is that the handedness (a genetic factor) and the side of the road that the participants normally drive on (a cultural factor) are important influential factors in path selection. The handedness is considered to be the main influencing factor with the driving side modifying the impact of handedness. Thus, if the driving side is the same as the handedness, these factors reinforce each other however, if they are opposite, they work in opposition. Thus it is important to consider the handedness of an individual and the country from where they come when attempting to predict wayfinding behavior. These results are considered statistically significant. The length of the first leg of the path also had a considerable influence on an individual's path choice. The shortest leg first criterion was found to be more influential than the longest leg first criterion. This contradicts earlier urban wayfinding studies and is thought to be due to both the differences in the environments and the small and selective sample used in the urban studies. Furthermore, the path directness does not appear to be a significant path selection criterion based on the results of the wayfinding tasks.

By considering the responses to the wayfinding tasks, the participant's preference for right-handed, lefthanded, SLF or LLF paths was determined. The preferences for these paths for the entire sample were found to be: $31 \%$ prefer right-handed paths, $29 \%$ prefer SLF paths, $23 \%$ prefer LLF paths and $17 \%$ prefer left-handed paths. This distribution provides a means for ranking path preference within evacuation model wayfinding algorithms. However, it is acknowledged that not every individual within a given population will exhibit the same type of behavior as suggested by the average preference distribution for 
the entire sample. Furthermore, there are significant differences in the wayfinding preferences of important sub-populations as genetic e.g. handedness, and cultural e.g. driving side, differences influence wayfinding preferences. Thus, the preference distribution for the factors influencing wayfinding was further refined to reflect handedness and driving side. It is not clear how large an effect these sub-population differences in path preference criteria will have on evacuation analysis. This is currently being examined by introducing these factors into a new wayfinding algorithm recently introduced into the buildingEXODUS evacuation model. Finally, when reviewing these findings it must be noted that the study is a map based questionnaire and so relies on the ability of participants to imagine themselves in the described situation. It is not clear if the participants would behave in a similar manner in a real emergency evacuation situation.

\section{REFERENCES}

[1] Kuligowski, E.D, The SFPE Handbook of Fire Protection Engineering, Quincy, MA, 2008, pp. 3456-3-478.

[2] Xie, H., Filippidis, L., Galea, E.R., Blackshields, D. and Lawrence, P.J., "Experimental Study of the Effectiveness of Emergency Signage," Fourth International Symposium of Human Behaviour in Fire, Interscience Communications, 2009, pp. 289-300.

[3] Filippidis, L., Lawrence, P.J., Galea, E.R. and Blackshields, D., (2008) Simulating the Interaction of Occupants with Signage systems, Fire Safety Science 9: 389-400, http://dx.doi.org/10.3801/IAFSS.FSS.9-389

[4] Veeraswamy, A., Lawrence, P.J. and Galea, E.R., "Implementation of Cognitive Mapping, Spatial Representation and Wayfinding Behaviours of People within Evacuation Modelling Tools," Fourth International Symposium of Human Behaviour in Fire, Interscience Communications, 2009, pp. 501-512.

[5] Golledge, R. G., "Path Selection and Route Preference in Human Navigation - A Progress Report," The University of California Transportation Center, UCTC No. 277, Berkeley, 1995, pp. $1-17$.

[6] Golledge, R.G., "Defining the Criteria Used in Path Selection," The University of California Transporation Center, UCTC No.278, Berkeley, 1995, pp. 1-23.

[7] Conroy, R.D., (2003) The secret is to follow your nose: Route path selection and angularity, Environment and Behavior 35(1): 107-131, http://dx.doi.org/10.1177/0013916502238867

[8] Ramadier, T. and Moser, G., (1998) Social Legibility, The Cognitive Map and Urban Behavior, Journal of Environmental Psychology 18: 307-319, http://dx.doi.org/10.1006/jevp.1998.0099

[9] Scharine, A. A. and McBeath, M, K., (2002) Right-Handers and Americans Favor Turning to the Right, Human Factors and Ergonomics Society 44(1): 248-256, http://dx.doi.org/10.1518/0018720024497916

[10] Raymond, M., Pontier, D., Dufour, A.B. and Moller, A.P., (1996) Frequency-dependent maintenance of left handedness in humans, Biological Sciences 263(1377): 1627-1633, http://dx.doi.org/10.1098/rspb.1996.0238

[11] Yates, F., (1934) Contingency Tables Involving Small Numbers and the $\chi^{2}$ Tests, Journal of the Royal Statistical Society 1(B): 217-235, http://dx.doi.org/10.2307/2983604

[12] Cochran, W.G., (1952) The $\chi^{2}$ Test of Goodness of Fit, Annals of Mathematical Statistics 23: 315345, http://dx.doi.org/10.1214/aoms/1177729380 